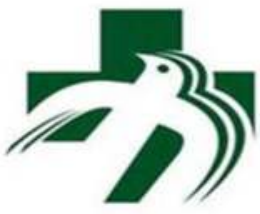

北京流和琵院

PEKING UNION MEDICAL. COLLEGE HOSPITAL

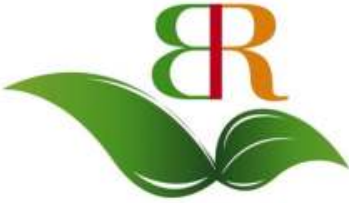

江苏博润集团

JIANGSU BO-RUN GROUP

\title{
Is suction blister epidermal grafting a simple and reliable way to screen patients with large area vitiligo for ReCell treatment?
}

AUTHORS: Zhifei Liu ${ }^{1}$, Weiwei $\mathrm{Li}^{2}$, Ang Zeng ${ }^{1}$, Zhongping. Meng ${ }^{3}$,Shunming Cheng ${ }^{3}$,Chunyu $\mathrm{Xu}^{4}$, Yong $\mathrm{Tao}^{5}$, Zhenxing Shen ${ }^{6}$, Shuzhen Zhang ${ }^{7},{\text { Zuomei } \mathrm{Li}^{7}}^{7}$ INSTITUTIONS : 1. PUMC hospital, Beijing, China. 2. Tsinghua Changgung hospital, Beijing, China. 3. Borun vitiligo hospital, Chengdu, China. 4. Shengjing vitiligo hospital, Haerbin, China. 5. Huaxia Vitiligo hospital, Nanjing, China. 6. Xinjundu vitiligo hospital, Urumuqi, China. 7. Kunming vitiligo hospital, Kunming, China.

\section{Introduction}

ReCell treatment (autologous non-cultured epidermal cell suspension grafting, ANECSG) is a method of choice for surgical treatments for large area and stable vitiligo, but not all ReCell treatments are effective and not all patients are fit for the treatment. Thus, a simple and reliable way to screen those suitable for ReCell treatment is urgently necessary and of important significance.

\section{Objective}

to explore if suction blister epidermal grafting (SBEG) is a simple and reliable way to screen patients with large area vitiligo for ReCell treatment.

\section{Materials and Methods}

49 patients with large area and stable vitiligo (lesion area $\geq 150 \mathrm{~cm} 2$, stable for $\geq 6$ months) were selected. Among which, 36(G1) have had successful SBEG, 13(G2) havn't had SBEG before ReCell treatments. All lesion areas were dermabraded and treated with ANECSG. Phototherpies (Excimer laser, wavelength of $308 \mathrm{~nm}$ ) start 15 days after surgery, continue twice a week for 3 consecutive months. Repigmentation rate was evaluated at 6 months after ReCell treatment. Results were graded as excellent, good, fair and poor according to repigmentation rate $>90 \%$, between $71 \%$ $90 \%, 51 \%-70 \%$ and $\leq 50 \%$, respectively

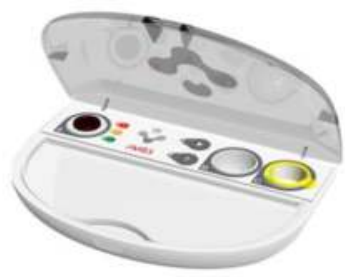

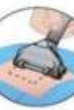
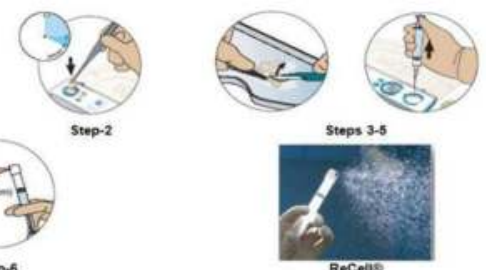
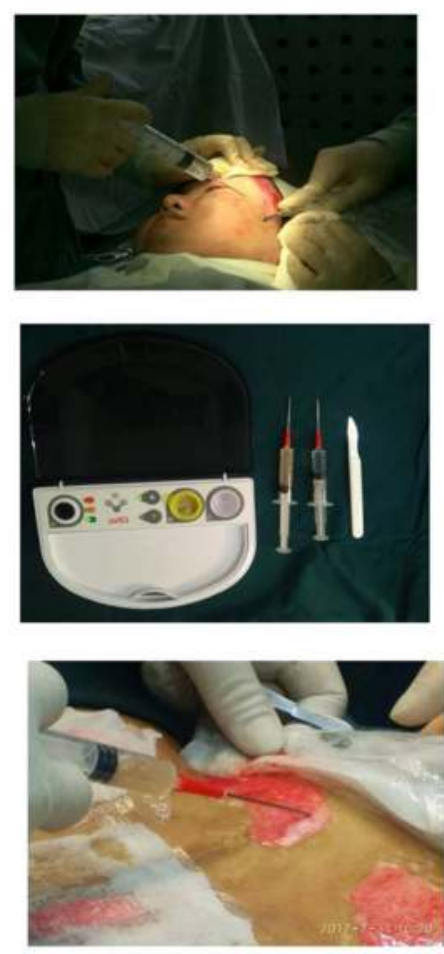

Fig 2 Recell kit treating procedure

Results

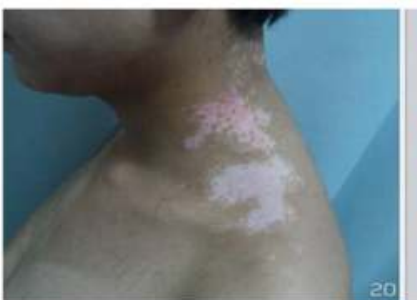

Pre-OP

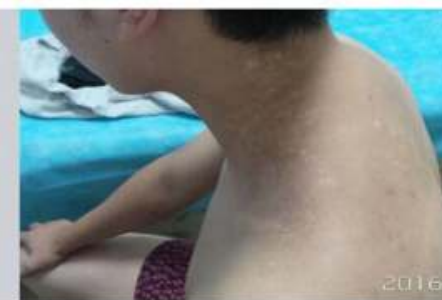

3 months Post SBEG

Fig 3 Screen patient with SBEG

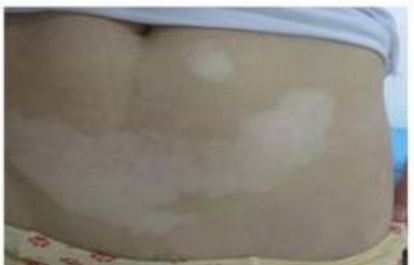

Pre-OP

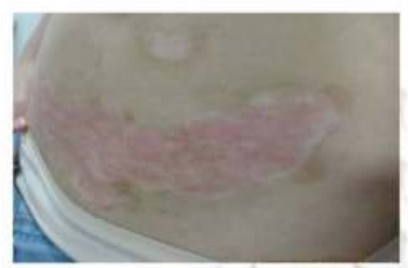

3 months post-Recell

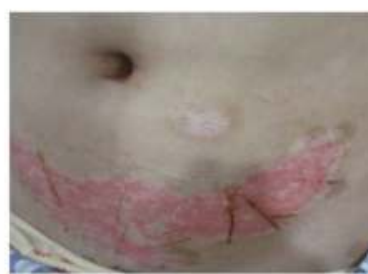

7 days post-Recell

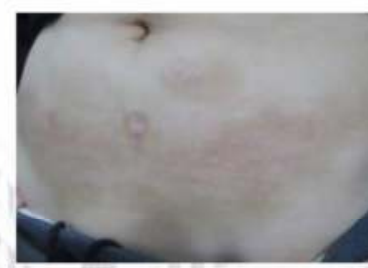

6 months post-Recell

Fig 4 Comparison of the vitiligo after Recell treatment

Results: In G1,18 patients ( $50 \%$ ) were excellent, $13(36.6 \%)$ were good, $5(13.9 \%)$ were fair, none were poor. In G2, 2(15.4\%) were excellent, $3(23.1 \%)$ were good, $4(30.7 \%)$ were fair, and $4(30.7 \%)$ were poor. Total repigmentation rates $\geq 71 \%$ and $>90 \%$ were both significantly higher in $\mathrm{G} 1$ than that in $\mathrm{G} 2(\mathrm{P}<0.01$, $\mathrm{P}<0.05$ ). None in 2 groups presented complications such as infection and scarring.

\section{Conclusion}

SBEG is a simple and reliable way to screen patients with large area and stable vitiligo for ReCell treatment.

\section{Reference}

1.O'Connor N, Mulliken J, Banks-Schlegel S, et al Grafting of burns with cultured epithelium prepared from autologous epidermal cells.[J]. Lancet, 1981, 1(8211):75-8

2. Sood R, Roggy D E, Zieger M J, et al. A comparative study of spray keratinocytes and autologous meshed split-thickness skin graft in the treatment of acute burn injuries.[J]. Wounds A Compendium of Clinical Research \& Practice, 2015, 27(2):31-40.

3.Cervelli V1, De Angelis B, Spallone D, et al. Use of a novel autologus cell-harvesting device to promote epithelialization and enhance appropriate pigmentation in scar reconstruction. Clin Exper Dermatol, 2009, 35: 776-780. 